\title{
Heparin Reduces Neuroinflammation and Transsynaptic Neuronal Apoptosis in a Model of Subarachnoid Hemorrhage
}

\author{
J. Marc Simard • Cigdem Tosun • Svetlana Ivanova • \\ David B. Kurland • Caron Hong • Leanne Radecki • \\ Carter Gisriel • Rupal Mehta • David Schreibman • \\ Volodymyr Gerzanich
}

Received: 16 February 2012 /Revised: 27 March 2012 / Accepted: 29 March 2012 / Published online: 14 April 2012

(C) The Author(s) 2012. This article is published with open access at Springerlink.com

\begin{abstract}
Subarachnoid hemorrhage (SAH) can lead to disabling motor, cognitive, and neuropsychological abnormalities. Part of the secondary injury to cerebral tissues associated with SAH is attributable to the neuroinflammatory response induced by blood. Heparin is a pleiotropic compound that reduces inflammatory responses in conditions outside the central nervous system. Using a model of SAH devoid of global insult, we evaluated the effect of delayed intravenous (IV) infusion of heparin, at a dose that does not produce therapeutic anticoagulation, on neuroinflammation, myelin preservation, and apoptosis. Adult male rats underwent bilateral stereotactic injections of autologous blood $(50 \mu \mathrm{L})$ into the subarachnoid space of the entorhinal cortex. The rats were implanted with mini-osmotic pumps that delivered either vehicle or unfractionated heparin $(10 \mathrm{U} / \mathrm{kg} / \mathrm{h} \mathrm{IV}$ ) beginning $12 \mathrm{~h}$ after SAH. No mechanical or
\end{abstract}

J. M. Simard $(\bowtie) \cdot$ C. Tosun $\cdot$ S. Ivanova $\cdot$ D. B. Kurland •

L. Radecki $\cdot$ C. Gisriel $\cdot$ V. Gerzanich

Department of Neurosurgery,

University of Maryland School of Medicine,

22 S. Greene St., Suite S12D,

Baltimore, MD 21201-1595, USA

e-mail: msimard@smail.umaryland.edu

J. M. Simard $\cdot$ R. Mehta

Department of Pathology,

University of Maryland School of Medicine,

Baltimore, MD, USA

J. M. Simard

Department of Physiology,

University of Maryland School of Medicine,

Baltimore, MD, USA

C. Hong $\cdot$ D. Schreibman

Department of Anesthesiology,

University of Maryland School of Medicine,

Baltimore, MD, USA hemorrhagic injury was observed in the hippocampus. In vehicle controls assessed at $48 \mathrm{~h}, \mathrm{SAH}$ was associated with robust neuroinflammation in the adjacent cortex [neutrophils, activated phagocytic microglia, nuclear factor-kappa $\mathrm{B}$, tumor necrosis factor-alpha, and interleukin-1beta] and neurodegeneration (Fluoro-Jade $\mathrm{C}$ staining and loss of NeuN). In the hippocampus, a muted neuroinflammatory response was indicated by Iba1-positive, ED1-negative microglia exhibiting an activated morphology. The perforant pathway showed FluoroJade C staining and demyelination, and granule cells of the dentate gyrus had pyknotic nuclei, labeled with Fluoro-Jade C and showed upregulation of cleaved caspase-3, consistent with transsynaptic apoptosis. Administration of heparin significantly reduced neuroinflammation, demyelination, and transsynaptic apoptosis. We conclude that delayed IV infusion of low-dose unfractionated heparin may attenuate adverse neuroinflammatory effects of SAH.

Keywords Subarachnoid hemorrhage .

Neuroinflammation · Microglia $\cdot$ Heparin $\cdot$ Transsynaptic apoptosis $\cdot$ Rat

\section{Introduction}

Subarachnoid hemorrhage (SAH) comprises 5-7\% of all strokes $[30,38]$. Acutely, $30 \%$ or more of patients die from the initial hemorrhage or rehemorrhage [30, 38]. Patients who survive are at high risk for potentially severe neurological and neuropsychological complications that develop during the subsequent days and weeks, with well over half succumbing to cerebral vasospasm, stroke, hydrocephalus, or cognitive and neuropsychological deficits. Among the worst cases are those in which the hemorrhage involves the eloquent cortex, a situation frequently encountered with 
rupture of middle cerebral artery aneurysms $[55,56]$. However, even among patients with mild hemorrhages (World Federation of Neurosurgical Societies grade 1) who do not suffer clinical or radiological vasospasm and who do not experience perioperative complications, long-term psychosocial and cognitive difficulties are still very common $[6,10]$.

Collectively, the various neurological and neuropsychological abnormalities brought on by SAH may be referred to as "delayed neurological deficits" (DNDs) [74]. Historically, the most devastating cause of DNDs was considered to be vasospasm leading to ischemia and stroke. Recently, a large clinical trial demonstrated that successful prevention of angiographic vasospasm by inhibition of endothelin signaling does not translate into improved clinical outcomes [48]. This landmark finding indicates that other mechanisms of secondary injury induced by blood, possibly unrelated to ischemia, play a crucial role in the development of DNDs [5, $8,21,31]$.

SAH has long been known to induce an inflammatory response in blood vessels and neuronal tissues [11, 17, 24, $29,52,61,64,72,73,77,78,84]$. Blood incites a robust inflammatory response in the central nervous system that is attributable, in part, to oxidative stress [43]. Many conventional markers of inflammation are activated or upregulated by $\mathrm{SAH}$, including leukocytes, microglia, immune complexes, complement, C-reactive protein, and numerous cytokines and chemokines [11, 22, 24, 37, 62, 73, 77]. Despite convincing evidence for the involvement of inflammation, block of conventional inflammatory/immune pathways using a variety of agents (glucocorticoids, lazaroids, iron chelators, cyclosporine, tacrolimus, and nonsteroidal antiinflammatory drugs) has been of limited benefit in ameliorating DNDs associated with $\mathrm{SAH}$, both in animal models and in humans [11, 72]. Nevertheless, recent studies examining nonconventional anti-inflammatory agents have shown promise $[73,77]$.

Heparin is a member of a family of polyanionic polysaccharides called glycosaminoglycans, composed of hexuronic acid and D-glucosamine residues joined by glycosidic linkages [9]. Although clinically utilized almost exclusively as an anticoagulant, heparin binding can interrupt numerous biological pathways [45]. Heparin is a pleiotropic drug that has long been recognized to have broad anti-inflammatory and immunomodulatory activities that are independent of its anticoagulant effect $[18,44,45,65,80,86]$. On the extensive list of heparin-binding proteins are several important cytokines and all chemokines $[18,80,86]$.

We recently reviewed the potential benefits of heparin as a pleiotropic, prophylactic agent in the context of SAH, showing how heparin can potentially antagonize most of the pathophysiological mechanisms known to be activated following SAH [74]. Here, we evaluated the effect of delayed intravenous (IV) infusion of heparin, at a dose that does not produce therapeutic anticoagulation [4, 42], in a model of SAH involving the entorhinal cortex, the part of the temporal lobe that projects directly to the hippocampus via the perforant pathway. In this model, SAH was associated with robust neuroinflammation in the adjacent cortex and white matter, with muted neuroinflammation in the hippocampus, with demyelination of the perforant pathway, and with transsynaptic apoptosis in the dentate gyrus. Here, we report that delayed IV infusion of low-dose heparin significantly reduced neuroinflammation, demyelination, and transsynaptic apoptosis.

\section{Methods}

\section{Subjects and Experimental Series}

All experimental procedures were approved by the University of Maryland Institutional Animal Care and Use Committee. Male Wistar rats (300-350 g; Harlan, Indianapolis, IN, USA) were used in this study, which was comprised of two experimental series: In series 1, eight rats underwent bilateral SAH (see below), three rats underwent unilateral SAH (right side only), and three rats underwent sham injury [same procedure but with injection of normal saline (NS) instead of blood]. They were euthanized at $48 \mathrm{~h}$. These brains were used for pathological and histochemical characterization of the model, including assessment for infarction using 2,3,5-triphenyltetrazolium chloride (TTC) staining (three rats with bilateral SAH), assessment of tissue damage (Fluoro-Jade C staining) with saline vs. blood (three rats each with unilateral saline vs. blood), and evaluation of the hemorrhage location with hematoxylin and eosin (H\&E) staining (five rats with bilateral SAH). In series 2, 16 rats underwent bilateral SAH and were implanted with miniosmotic pumps that delivered either NS (8 rats) or heparin ( 8 rats; see below). These brains were used for further histochemical and immunohistochemical characterization of the model and to assess the effect of heparin treatment. In accordance with "good laboratory practice," surgical procedures were conducted by investigators blinded to treatment.

\section{Rat Model of SAH}

Anesthetized (ketamine $60 \mathrm{mg} / \mathrm{kg}$ and xylazine $7.5 \mathrm{mg} / \mathrm{kg}$ intraperitoneally) rats spontaneously breathed room air supplemented with oxygen to maintain $90 \%<\mathrm{SO}_{2}<98 \%$ by pulse oximetry. Temperature was maintained at $37^{\circ} \mathrm{C}$ using a heating pad regulated by a rectal temperature sensor (Harvard Apparatus, Holliston, MA, USA). Surgical procedures were carried out using aseptic techniques.

$\mathrm{SAH}$ was produced by injecting $50 \mu \mathrm{L}$ of autologous blood under stereotactic guidance into the subarachnoid 
space of the entorhinal cortex, bilaterally. First, the tail artery was cannulated (PE-20 tubing) under microscopic visualization. The head was fixed in a stereotaxic apparatus (Stoelting Co., Wood Dale, IL, USA) and a midline scalp incision was made. One burr hole was placed at (bregma and midline as reference points) anterior-posterior, $-7.7 \mathrm{~mm}$; lateral, $+5 \mathrm{~mm}$; a second burr hole was placed at anteriorposterior, $-7.7 \mathrm{~mm}$; lateral, $-5 \mathrm{~mm}$. The dura at each burr hole was pierced sharply. Then, $600 \mu \mathrm{L}$ of blood was aspirated from the tail artery; the last $125 \mu \mathrm{L}$ of nonheparinized blood was aspirated into a syringe equipped with a 22-mm-long blunt, tapered-tip needle (Hamilton custom tapered syringe, 7732-01; Hamilton Co., Reno, NV, USA). The syringe was mounted in the stereotaxic apparatus and the tip coordinates were registered. Beginning at the burr hole on the right side, the needle was advanced to the $z$-coordinate of -7.0 , then was advanced very slowly until the curvature of the posterior aspect of the middle fossa was reached, as indicated by the needle shifting slightly anteriorly [at the $z$-coordinate (mean \pm $\mathrm{SD}),-8.3 \pm 0.4 \mathrm{~mm}$; range, -7.3 to $-9.2 \mathrm{~mm}$ ]. Use of a blunt, tapered-tip needle prevented the dura and bone from being pierced, allowing reliable access to the subarachnoid space. Without adjusting the depth, $50 \mu \mathrm{L}$ of blood was injected over $5 \mathrm{~min}$, after which $5 \mathrm{~min}$ was allowed to elapse before slowly withdrawing the needle. The procedure was repeated on the left side.

\section{Treatment}

In rats that would be administered drug, IV access to the external jugular vein was obtained under microscopic visualization. A mini-osmotic pump (1.0 $\mu \mathrm{L} / \mathrm{h}$; Azlet 2001, Durect Corporation, Cupertino, CA, USA) fitted with a rat jugular vein catheter (\#0007710, Alzet, Durect Corporation, Cupertino, CA, USA) was implanted. The catheter was filled sequentially with $11 \mu \mathrm{L}$ of NS, $1 \mu \mathrm{L}$ of air, and the remainder with the solution to be administered; the first 12 $\mu \mathrm{L}$ in the catheter provided a $12-\mathrm{h}$ delay in the start of drug administration [75]. The pumps were filled either with NS or with heparin sodium (5,000 USP U/ml; APP Pharmaceuticals, LLC, Schaumburg, IL, USA) in NS (1 ml of heparin stock plus $0.43 \mathrm{ml} \mathrm{NS}$ ) that delivered $10 \mathrm{U} / \mathrm{kg} / \mathrm{h}$. Therapeutic anticoagulation in the rat requires constant infusion of $75 \mathrm{U} / \mathrm{kg} / \mathrm{h}$ of heparin [42].

\section{Histochemistry}

After transcardiac perfusion/fixation with $10 \%$ neutral buffered formalin, the brains were cryoprotected with $30 \%$ sucrose. For routine histology, cryosections $(10 \mu \mathrm{m})$ were stained with H\&E and examined using light microscopy.

To assess myelin, cryosections $(10 \mu \mathrm{m})$ were stained with Black Gold II according to manufacturer's protocol (Black
Gold II Myelin Staining Kit, AG105, Millipore, Temecula, CA, USA). Briefly, slides were first rehydrated and incubated in prewarmed $0.3 \%$ Black Gold II solution at $60^{\circ} \mathrm{C}$ for $15 \mathrm{~min}$. Sections were then rinsed twice, and then incubated in $1 \%$ sodium thiosulfate solution for $3 \mathrm{~min}$ at $60^{\circ} \mathrm{C}$. After several rinses with Milli-Q water, the sections were counterstained with cresyl violet to visualize nuclei, were coverslipped, and were examined using light microscopy.

To identify degenerating neurons and white matter tracts, cryosections $(10 \mu \mathrm{m})$ were labeled with Fluoro-Jade C histofluorescent stain [70] according to the manufacturer's protocol (Histo-Chem, Jefferson, AR, USA). Sections were coverslipped with nonpolar mounting medium (Cytoseal XYL, Richard-Allan Scientific, Kalamazoo, MI, USA) and were examined with an epifluorescence microscope (Nikon Eclipse 90i) under a fluorescein isothiocyanate filter, with care being taken to limit the time of exposure in order to reduce photobleaching.

\section{Immunohistochemistry}

Cryosections $(10 \mu \mathrm{m})$ were first blocked (5 \% goat serum, Sigma $+0.2 \%$ Triton $\mathrm{X}-100$ for $1 \mathrm{~h}$ at room temperature) and then incubated overnight at $4{ }^{\circ} \mathrm{C}$ with the following primary antibodies: rabbit anti-ionized calcium-binding adapter molecule 1 (Iba1) $(1: 1,000$; Wako Chemicals, Richmond, VA, USA); mouse anti-ED-1 (1:200; MAB1435; Millipore, Temecula, CA, USA); rabbit antimyeloperoxidase (1:200; A0398; Dako, Carpinteria, CA, USA); rabbit antinuclear factor-kappa B (p65) (1:200; sc-372; Santa Cruz Biotechnology, Santa Cruz, CA, USA); rabbit anticleaved caspase-3 (1:200; Asp175; Cell Signaling Technologies, Beverly, MA, USA); mouse antiglial fibrillary acidic protein (GFAP) (1:500; CY3 conjugated; C-9205; Sigma, St. Louis, MO, USA); goat antitumor necrosis factor-alpha $(\mathrm{TNF} \alpha)(1: 100 ; \mathrm{N}-19$, \#SC-1350; Santa Cruz Biotechnology, Santa Cruz, CA, USA); rabbit anti-interleukin-1beta (IL-1 $\beta$; \#AB9787; Abcam, Cambridge MA, USA).

After several rinses in phosphate-buffered saline, the slides were incubated for $1 \mathrm{~h}$ with fluorescent-labeled species-appropriate secondary antibodies (1:500; Alexa Flour 488 and Alexa Flour 555; Invitrogen, Molecular Probes, Eugene, OR, USA) at room temperature. Omission of primary antibody was used as a negative control. The sections were coverslipped with polar mounting medium containing antifade reagent and 4',6-diamidino-2-phenylindole (DAPI; Invitrogen, Eugene, OR, USA) and were examined using epifluorescence microscopy (Nikon Eclipse 90i; Nikon Instruments Inc., Melville, NY, USA). In some cases, for the sake of clarity of presentation, epifluorescence images are presented in the figures as "inverse" images, 
wherein the brightness value of each pixel is converted to the inverse value on a 256-step scale.

\section{Quantitative Immunohistochemistry}

Quantifications comparing the effect of vehicle vs. heparin were made using the right side of the brain. Unbiased measurements of specific labeling within regions of interest (ROI) were obtained using NIS-Elements AR software (Nikon Instruments, Melville, NY, USA) from sections immunolabeled in a single batch, as previously described $[26,60]$. All ROI images for a given signal were captured using uniform parameters of magnification, area, exposure, and gain. The following ROIs were defined: (1) for MPO, $\mathrm{ED} 1, \mathrm{p} 65, \mathrm{TNF} \alpha$, and IL-1 $\beta$ in the entorhinal cortex, a rectangular ROI, $1 \times 1 \mathrm{~mm}$, that was positioned on the pial surface; (2) for Iba1 in the hippocampus, a rectangular ROI that was positioned on the perforant pathway (see Fig. 6); (3) for Black Gold II in the hippocampus, a rectangular ROI that was positioned in the region between the perforant pathway and the dentate gyrus (see Fig. 7); (4) for FluoroJade $\mathrm{C}$ in the hippocampus, a rectangular ROI, $450 \times$ $360 \mu \mathrm{m}$, that was positioned at the hilus of the dentate gyrus. Segmentation analysis was performed by computing a histogram of pixel intensity for a particular ROI. Specific labeling was defined as pixels with signal intensity greater than two times that of background. For MPO, ED1, and p65, we performed object counts for specific labeling, which for MPO and ED1 corresponded to the number of cells. For Iba1 and Black Gold II, the area occupied by pixels with specific labeling was used to determine the percent area with specific labeling (\%ROI). For Fluoro-Jade C, we counted the number of neurons within the ROI.

\section{Statistical Analysis}

Calculations were performed with OriginPro8 (OriginLab Corp., Northampton, MA, USA). Statistical comparisons were made using Student's $t$ test or a one-way analysis of variance with Fisher's post hoc comparison, as appropriate.

\section{Results}

\section{SAH Involving the Entorhinal Cortex}

Using the methods described, we were able to take advantage of the natural curvature of the calvarium to reproducibly locate the subarachnoid space over the posterolateral temporal lobe and to produce a SAH that accumulated over the entorhinal cortex (Fig. 1a). The technique used, including the amount of blood and its slow injection, did not result in an ischemic injury to the region (Fig. 1b). Blood was
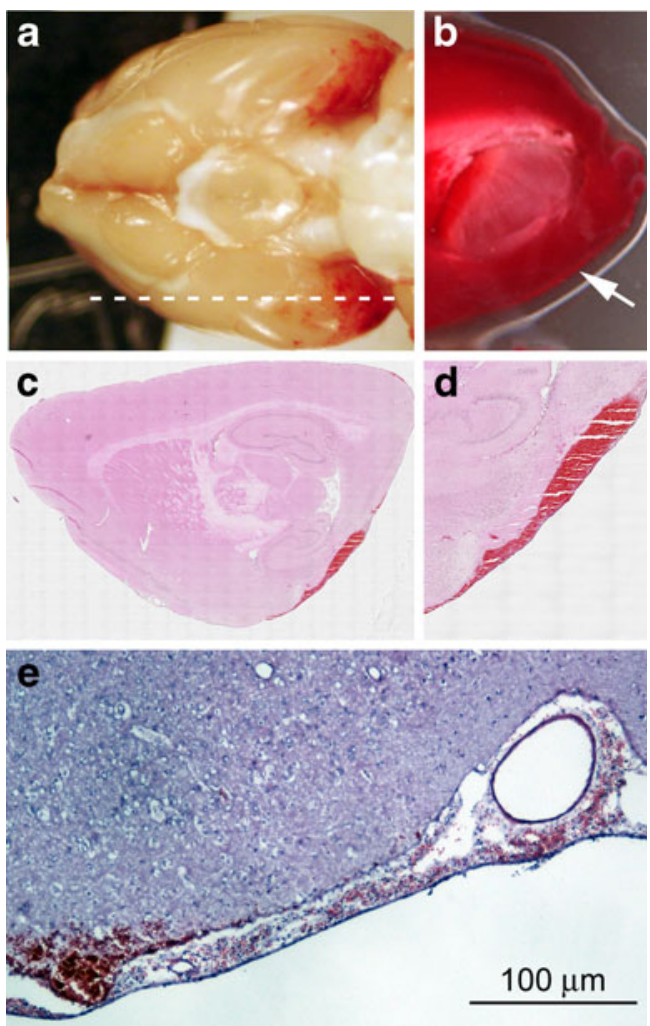

Fig. 1 SAH involving the entorhinal cortex. a Image of the base of the brain showing SAH involving the entorhinal cortex bilaterally; dotted line location of parasagittal sections. b Parasagittal section of the brain stained with TTC showing the absence of infarction in the region of SAH (arrow). c-e Parasagittal sections of the brain in the region of SAH stained with H\&E showing that the hemorrhage is localized largely in the subarachnoid space and that subarachnoid vessels show no evidence of vasoconstriction or vasospasm. All tissues were harvested $48 \mathrm{~h}$ after injury; the images shown are representative of findings in 28 rats $(\mathbf{a}), 3$ rats $(\mathbf{b})$, or 5 rats $(\mathbf{c}-\mathbf{e})$

largely confined to the subarachnoid space (Fig. 1c-e). Vasoconstriction or vasospasm of vessels in the subarachnoid space was not observed (Fig. 1e). This model was associated with near-zero mortality, with the rare deaths being attributable to anesthesia or surgery.

\section{Neurodegeneration in the Entorhinal Cortex}

At $48 \mathrm{~h}$ after $\mathrm{SAH}$, the adjacent cortex and white matter, including numerous neuron-like pyramidal cells, stained heavily with Fluoro-Jade C, as did the ipsilateral but not the contralateral perforant pathway (Fig. 2a, c, d). Saline injection did not result in staining with Fluor-Jade $\mathrm{C}$ above background levels (Fig. 2b). The cortical region that stained with Fluoro-Jade C showed downregulation of GFAP, but the surrounding tissues showed reactive astrocytosis (Fig. 2c, e). Compared to the control, the cortical region that stained with Fluoro-Jade $\mathrm{C}$ was depleted of NeuNpositive cells (Fig. 2f, g). 

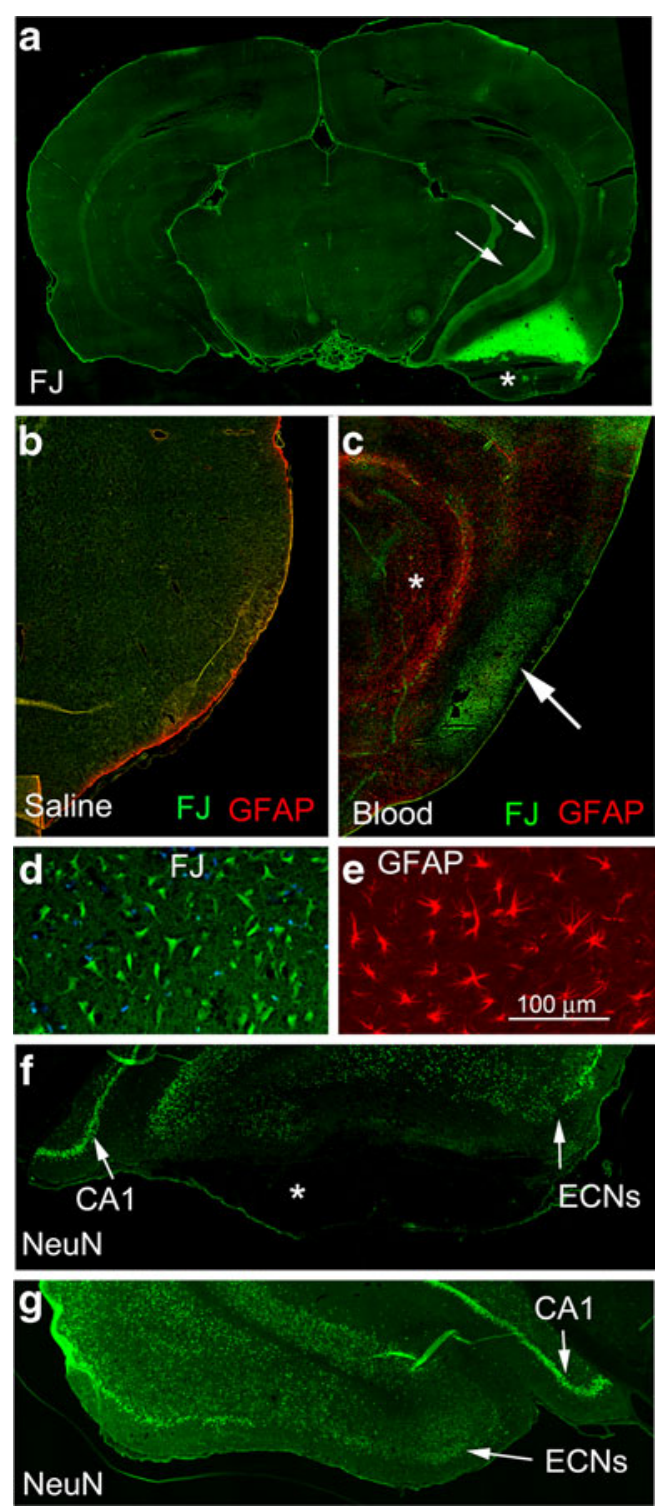

Fig. 2 Cortical neurodegeneration following SAH. a Coronal section from a rat with unilateral injection of $50 \mu \mathrm{L}$ of autologous blood into the subarachnoid space of the entorhinal cortex, stained with FluoroJade $\mathrm{C}(F J)$; note the bright staining above the background in the cortex and underlying white matter near the hemorrhage, along with the bright staining of the perforant pathway ipsilateral (arrows) but not contralateral to the hemorrhage; asterisk denotes hemorrhage. b-e Parasagittal sections of rat brains in the region of injection of saline (b) or blood (c-e), stained with Fluoro-Jade C (b-d) and immunolabeled for GFAP (b, c, e) showing degeneration involving neuron-like cells in the cortex beneath the SAH (c, d), but not with saline (b) and reactive astrocytes surrounding the region that stains for Fluoro-Jade $\mathrm{C}$ (c, e). f, $\mathbf{g}$ Coronal sections from a rat with unilateral injection of $50 \mu \mathrm{L}$ of autologous blood into the subarachnoid space of the entorhinal cortex, immunolabeled for NeuN, showing the entorhinal cortex ipsilateral (f) and contralateral (g) to the hemorrhage; note the loss of ipsilateral entorhinal cortex neurons $(E C N s)$ throughout the region, except laterally, and the preservation of neurons in the ipsilateral CA1 region and in all regions contralaterally $(\mathbf{g})$; asterisk denotes hemorrhage; same rat as in a. All tissues were harvested $48 \mathrm{~h}$ after injury; the images shown are representative of findings in five rats $(\mathbf{b}-\mathbf{e})$ or three rats $(\mathbf{a}, \mathbf{f}, \mathbf{g})$
Neuroinflammation in the Entorhinal Cortex

At $48 \mathrm{~h}$ after SAH, adjacent subpial tissues showed upregulation of the p65 subunit of nuclear factor-kappa B (NF-kB) in cells and microvessels, with $\mathrm{p} 65$ expression tapering off to normal within a few hundred micrometers (Fig. 3a, b). The subpial tissues were infiltrated with neutrophils and ED1-positive cells, consistent with macrophages and activated, phagocytic microglia (Fig. 3c). Immunolabeling for Iba1, which identifies all microglia regardless of their activation state, showed a gradient, from the pial edge to deeper, of cells with a rounded, activated morphology subpially that transitioned into a less activated morphology further away (Fig. 3d).

Effect of Heparin on Neuroinflammation in the Entorhinal Cortex

We examined the effect of heparin on counts of neutrophils, ED1-positive cells, and p65 upregulation in subpial tissues of the entorhinal cortex (Fig. 4). Immunolabeling for MPO showed that the subpial distribution of neutrophils appeared similar (Fig. 4a, b) and that counts of neutrophils were reduced, but not statistically different (Fig. 4e) in the two groups. Immunolabeling for ED1 showed that the "front" of ED1-positive cells appeared to extend less deeply (Fig. 4c, d) and that counts of ED1-positive cells were significantly reduced by heparin (Fig. 4e). Immunolabeling for p65 showed that upregulation of $\mathrm{p} 65$ was significantly reduced by heparin (Fig. 4e).

We also examined the effect of heparin on the expression of TNF $\alpha$ and IL-1 $\beta$ in subpial tissues of the entorhinal cortex (Fig. 5). Immunolabeling for TNF $\alpha$ showed that the "front" of TNF $\alpha$ was less prominent and extended less deeply with heparin (Fig. 5a, b, e). Similarly, the expression of IL-1 $\beta$ as well as the count of IL-1 $\beta$-positive cells were significantly reduced by heparin (Fig. $5 \mathrm{c}-\mathrm{e}$ ).

\section{Effect of Heparin on Neuroinflammation}

in the Hippocampus

Immunolabeling for MPO, ED1, and p65 showed that none of these markers of inflammation were present in the hippocampus (not shown). Nevertheless, there was evidence of microglial activation, based on immunolabeling for Iba1 (Fig. 6), which identifies microglia regardless of activation state. In contrast to the Iba1-positive, ED1-positive, large, rounded, phagocytic microglia without processes observed subpially in the entorhinal cortex, microglial activation in the hippocampus was more muted. In the hippocampus of vehicle-treated rats, Iba1-positive cells were numerous (Fig. 6a); also, the cells were plump but not rounded and the processes were often enlarged (Fig. 6c). In heparin- 

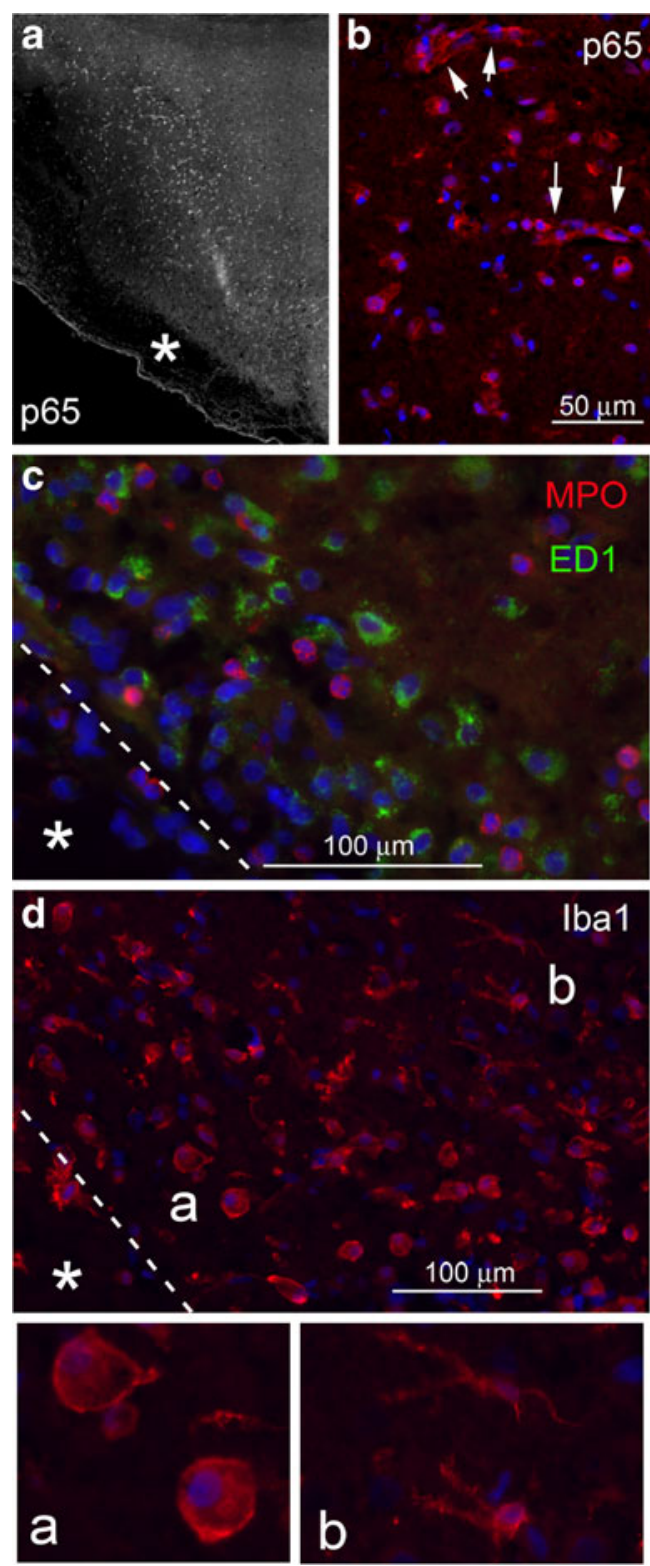

Fig. 3 Cortical neuroinflammation following SAH. a, b Parasagittal section immunolabeled for NF-kB (p65), shown at low (a) and high (b) magnification, demonstrating (1) prominent expression of p65 subpially near the hemorrhage (asterisk) that tapers off with distance (a); (2) involvement of individual cells as well as elongated structures consistent with microvessels (arrows) (b); the image shown in a is an "inverse" epifluorescence image. c Parasagittal section immunolabeled for myeloperoxidase (MPO) to identify neutrophils (note also the polymorphic nuclei) and ED1 to identify macrophages and phagocytic, activated microglia, showing tissue infiltration beneath the pia (dotted line). d Parasagittal section immunolabeled for Iba1, to identify microglia, showing (1) large rounded cells ( $a$ and inset $a$ ) beneath the pia (dotted line), consistent with the phagocytic, activated microglia labeled with ED1 in c; (2) small cells with complex processes further away from the hemorrhage ( $b$ and inset $b$ ). All tissues were harvested $48 \mathrm{~h}$ after injury; the images shown are representative of findings in five rats

treated rats, Iba1-positive cells were less prominent (Fig. 6b), the cell bodies were small, and the processes were finer

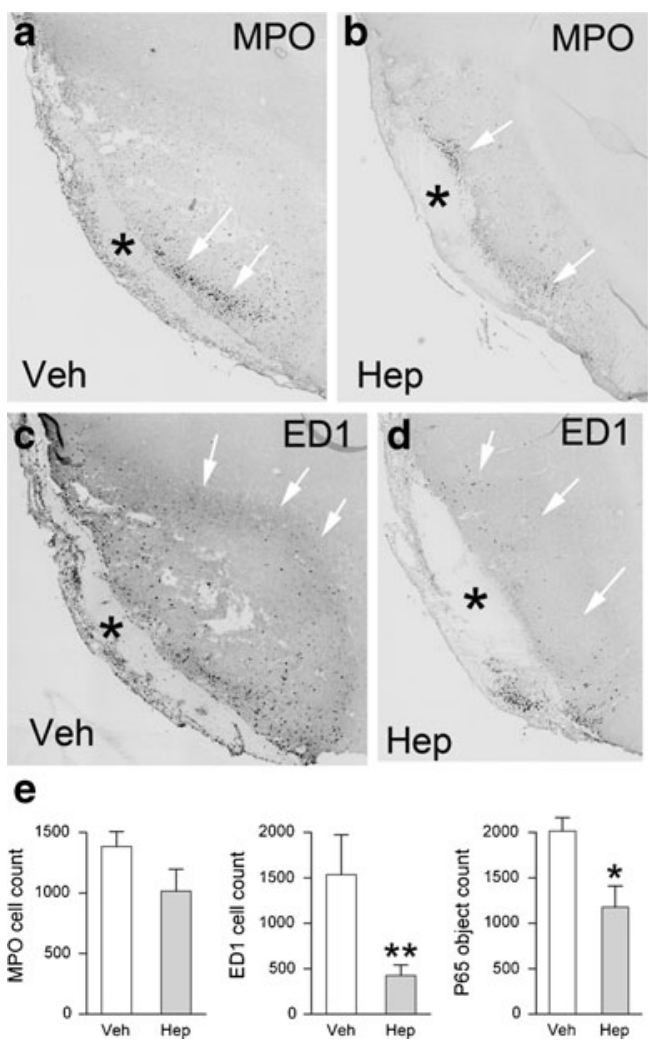

Fig. 4 Heparin reduces cortical neuroinflammation (microglial activation) following SAH. a-d Parasagittal sections immunolabeled for myeloperoxidase (MPO) to identify neutrophils (a, b) or ED1 to identify macrophages and phagocytic, activated microglia (c, d) from rats treated with vehicle $(\mathbf{a}, \mathbf{c})$ or heparin $(\mathbf{b}, \mathbf{d})$; the advancing front of neutrophils, depicted by arrows (a, b), was similar without and with heparin; the advancing front of ED1-positive macrophages and microglia, depicted by arrows (c, d), was reduced by heparin; asterisk denotes the location of the SAH; the images shown are "inverse" epifluorescence images. e Bar graphs showing counts of MPOpositive cells, ED1-positive cells, and upregulation of p65 in rats treated with vehicle $(\mathrm{Veh})$ or heparin $(\mathrm{Hep}) ;{ }^{*} P<0.05$; $* * P<0.01$; five rats per group

(Fig. 6d). Upregulation of Iba1 was significantly reduced by heparin (Fig. 6e).

Myelin is highly susceptible to injury by oxidative stress [76]. We evaluated myelin preservation by staining with Black Gold II. Myelin was visibly deficient in the perforant pathway and in the dentate gyrus of vehicle-treated rats (Fig. 7a, c), consistent with the previous observation of Fluro-Jade C staining of the perforant pathway (Fig. 2a). By contrast, myelin appeared to be better preserved in the perforant pathway and dentate gyrus of heparin-treated rats (Fig. 7b, d). Quantification of myelin in the region between the perforant pathway and the dentate gyrus showed that myelin was significantly better preserved with heparin (Fig. 7e).

In vehicle-treated rats, many granule cells of the dentate gyrus exhibited pyknotic nuclei that immunolabeled for cleaved caspase-3 (Fig. 8a) and that stained with Fluoro- 


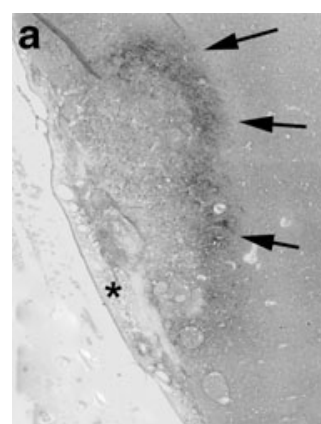

Veh TNF $\alpha$
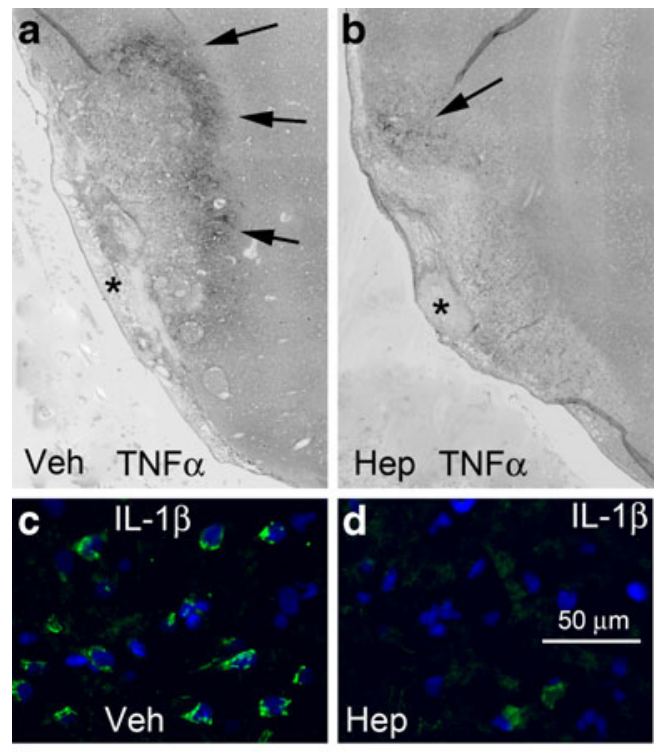

e
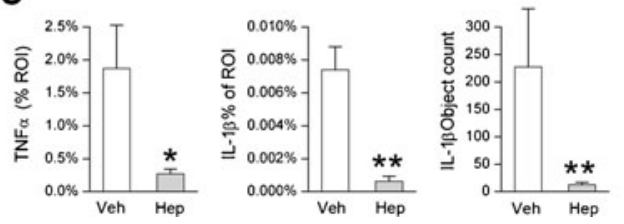

Fig. 5 Heparin reduces cortical neuroinflammation (TNF $\alpha$ and IL-1 $\beta$ ) following SAH. a-d Parasagittal sections immunolabeled for TNF $\alpha(\mathbf{a}$, b) or IL-1 $\beta(\mathbf{c}, \mathbf{d})$ from rats treated with vehicle $(\mathbf{a}, \mathbf{c})$ or heparin $(\mathbf{b}, \mathbf{d})$; the advancing front of TNF $\alpha$, depicted by arrows $(\mathbf{a}, \mathbf{b})$, was reduced by heparin; asterisk denotes the location of the $\mathrm{SAH}$; the images shown in $\mathbf{a}$ and $\mathbf{b}$ are "inverse" epifluorescence images. e Bar graphs showing the percentage of the ROI immunolabeled for TNF $\alpha$ or IL-1 $\beta$, as well as the number of cells immunolabeled for IL-1 $\beta$, as indicated, in rats treated with vehicle $(\mathrm{Veh})$ or heparin $(\mathrm{Hep}) ;{ }^{*} P<0.05 ;{ }^{* *} P<0.01$; five rats per group

Jade C (Fig. 8c). All Fluoro-Jade C-positive cells had pyknotic nuclei (Fig. 8e), also consistent with apoptosis. These findings were less prominent in rats treated with heparin (Fig. 8b, d). Quantification showed that FluoroJade C-positive cells were significantly reduced with heparin (Fig. 8f).

\section{Discussion}

We studied neuroinflammation in a model of SAH that involved the eloquent cortex and that was devoid of a global insult. To characterize microglial activation, we used two antibodies that convey complementary information. The ED1 antibody is a macrophage marker that recognizes an intracytoplasmic, lysosomal antigen whose expression increases during phagocytic activity in monocytes and other tissue macrophages, including microglia $[3,15,28]$. The Iba1 antibody is a marker directed against a microglia-
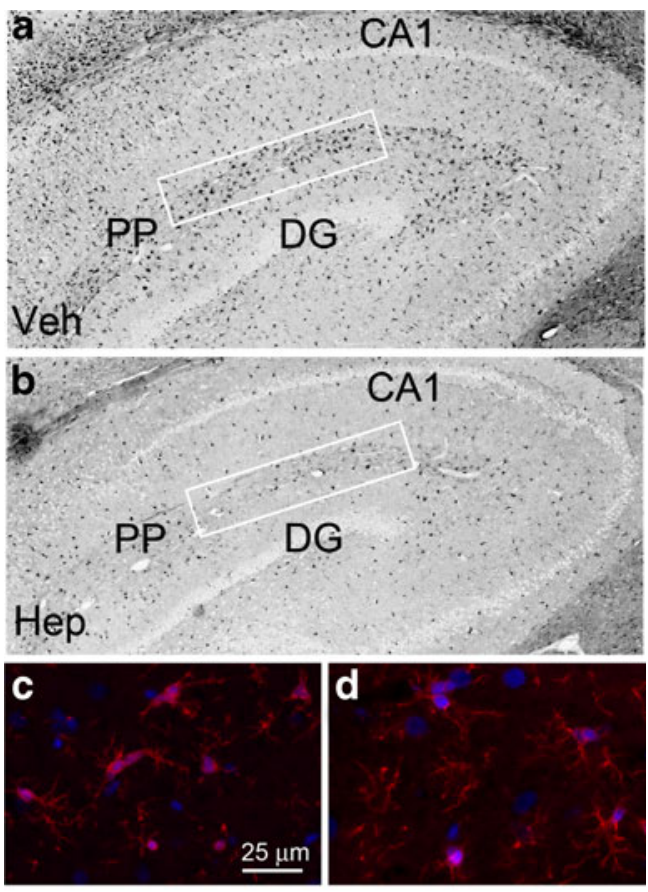

e

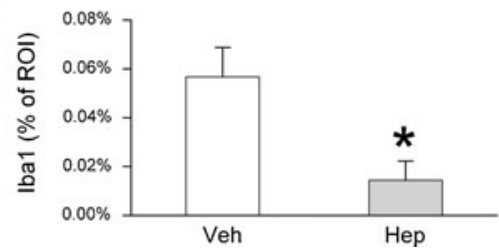

Fig. 6 Heparin reduces hippocampal neuroinflammation following SAH. a-d Parasagittal sections immunolabeled for Iba1, shown at low $(\mathbf{a}, \mathbf{b})$ and high $(\mathbf{c}, \mathbf{d})$ magnification, from rats treated with vehicle $(\mathbf{a}, \mathbf{c})$ or heparin $(\mathbf{b}, \mathbf{d})$; note the "plump" morphology of microglia in the controls (c), which is absent with heparin (d); CA1 cornu ammonis region 1, $P P$ perforant pathway, $D G$ dentate gurus; the ROI analyzed are depicted by rectangles; the images shown in $\mathbf{a}$ and $\mathbf{b}$ are "inverse" epifluorescence images. e Bar graph quantifying Ibal in the ROI in rats treated with vehicle $(\mathrm{Veh})$ or heparin $(\mathrm{Hep})$; ${ }^{*} P<0.05$; five rats per group

specific antigen that identifies nonactivated (resting), as well as activated but nonphagocytic microglia that are ED1negative [28]. Using these and other antibodies, we found a neuroinflammatory response in the entorhinal cortex adjacent to the hemorrhage that was robust, with invasion of neutrophils, ED1-positive cells, phagocytic microglia, and macrophages, and prominent labeling for NF-KB, TNF $\alpha$, and IL-1 $\beta[16,77]$. This robust neuroinflammatory response was associated with significant neurodegeneration, marked by Fluoro-Jade C staining and loss of NeuN labeling. The robust inflammatory response in the entorhinal cortex was very different from the muted inflammatory response observed remotely in the hippocampus, where there was no labeling for MPO, ED1, or NF- $\mathrm{kB}$ and where the principal evidence for neuroinflammation was the appearance of Iba1-positive microglia that exhibited an 


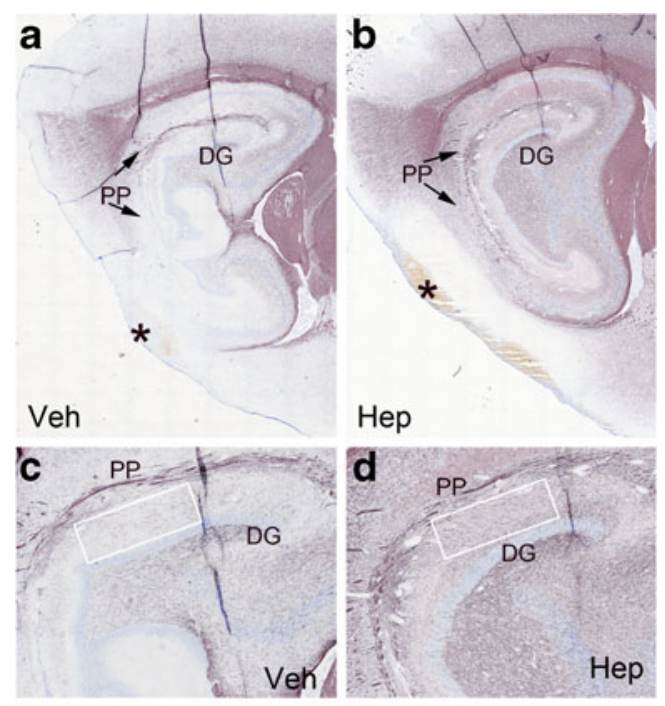

e

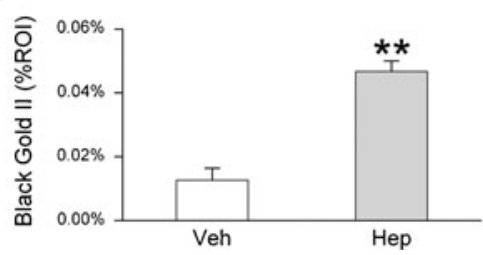

Fig. 7 Heparin reduces demyelination of the perforant pathway following SAH. a-d Parasagittal sections stained with Black Gold II, shown at low (a, b) and high (c, d) magnification, from rats treated with vehicle (a, c) or heparin (b, d); $P P$ perforant pathway, $D G$ dentate gyrus; the ROI analyzed is depicted by the rectangle. e Bar graph quantifying Black Gold II-stained myelin in the ROI in rats treated with vehicle $(\mathrm{Veh})$ or heparin $(\mathrm{Hep}) ; * * P<0.01$; five rats per group

activated morphology. Overall, our findings on neuroinflammation associated with SAH are consistent with previous reports $[2,11,17,24,29,52,61,64,72,73,77,78,84]$. What may be an importance difference, however, is that, in many of the previous reports, either the model used (endovascular puncture) or the amount of blood injected (200$500 \mu \mathrm{L}$ ) could potentially have been associated with a widespread or global ischemic insult, whereas in our experiments, the manner of injection and the amount of blood injected $(50 \mu \mathrm{L}$ on each side) were highly unlikely to produce an ischemic injury, as confirmed by the lack of vasoconstriction or vasospasm of vessels in the subarachnoid space and by uniformly positive tissue staining with TTC.

The major finding of the present study is that delayed IV infusion of heparin, at a dose that does not produce therapeutic anticoagulation, significantly reduced neuroinflammation associated with $\mathrm{SAH}$, both the proximate effect in the entorhinal cortex, as well as the remote effect in the hippocampus. Heparin is a pleiotropic drug that has long been recognized to have broad anti-inflammatory and immunomodulatory activities that are independent of its anticoagulant effect $[18,44,45,65,80,86]$. More than 100 heparin-binding proteins are known [86], with the growing
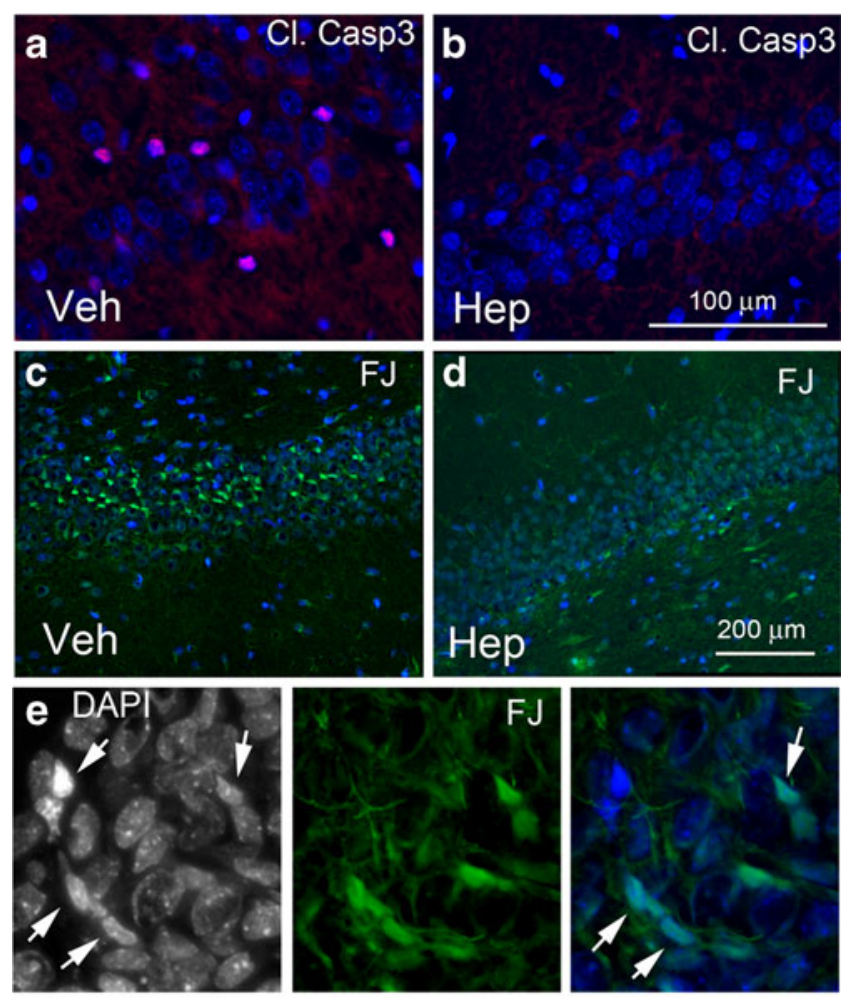

f

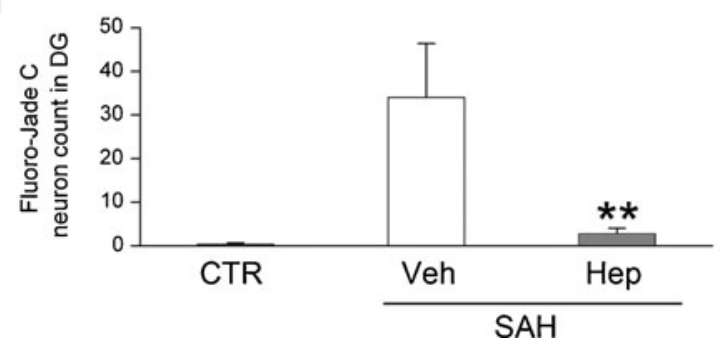

Fig. 8 Heparin reduces apoptosis of granule cells of the dentate gyrus following SAH. a-d Parasagittal sections of the dentate gyrus immunolabeled for cleaved caspase-3 (a, b) or stained with Fluoro-Jade C (c, d) from rats treated with vehicle $(\mathbf{a}, \mathbf{c})$ or heparin $(\mathbf{b}, \mathbf{d})$. e Granule cells of the dentate gyrus from a vehicle-treated rat, double stained with DAPI and with Fluoro-Jade C $(F J)$, as indicated, showing pyknotic nuclei of apoptotic cells (arrows); superimposed images are also shown. f Bar graph quantifying Fluoro-Jade C-stained granule cells in the dentate gyrus of naïve (uninjured) rats $(C T R)$, rats with bilateral $\mathrm{SAH}$ treated with vehicle $(\mathrm{Veh})$ or heparin $(\mathrm{Hep}) ; * * P<0.01$; three naïve rats and eight rats per group with $\mathrm{SAH}$

list including numerous plasma proteins, proteins released from platelets, cytokines, chemokines, and other small, biologically active molecules, as well as endothelial cells themselves [14, 25, 32, 36, 51, 65]. The anti-inflammatory effects of heparin are due to a number of interactions: (1) inhibition of complement activation [23, 83]; (2) binding to the leukocyte adhesion molecules, P- and L-selectin [65, 81]; (3) inhibition of the cationic neutrophil proteases, human leukocyte elastase, and cathepsin G [23, 65]; (4) disruption of Mac-1 (CD11b/CD18)-mediated leukocyte adhesion to the receptor for advanced glycation end 
products (RAGE) [65]; (5) inhibition of RAGE ligation by its many proinflammatory ligands, including the advanced glycation end product, carboxymethyl lysine-bovine serum albumin, the nuclear protein, high mobility group box protein-1, and S100 calgranulins [46, 47, 53, 65, 67]; (6) inhibition of the nuclear translocation of NF- $\mathrm{kB}$ and a decrease in NF-kB-DNA binding in the endothelium [79].

A recent comprehensive review showed that heparin exhibits a broad diversity of biological effects, many of which can be directly linked to pathophysiological mechanisms that have been implicated in SAH-induced DNDs [74]. Among its wide-ranging effects, heparin (1) complexes with free hemoglobin, including oxyhemoglobin [1]; (2) blocks the activity of free radicals including reactive oxygen species [20]; (3) antagonizes endothelin-mediated vasoconstriction $[12,41,85]$; (4) accelerates antithrombin-IIImediated degradation of thrombin [57] which is implicated in protracted vasoconstriction [35]; (5) binds to several growth factors, thereby imparting antimitogenic [33, 34, 39] and antifibrotic [66] effects; and as reviewed above, (6) exerts potent multitargeted anti-inflammatory effects. The efficacy reported here for heparin against neuroinflammation, combined with the fact that the dose we used was less than that required for therapeutic anticoagulation [4, 42], suggests that heparin may be a safe prophylactic agent for reducing DNDs associated with SAH.

Despite the absence of a global insult, we identified extensive cell degeneration and cell death in this model, especially in the entorhinal cortex and white matter near the SAH. Hippocampal and cortical cell death and neuronal apoptosis have been previously documented, both in models of SAH and in humans with SAH [7, 54], but the trigger for apoptosis is not always known. With the endovascular perforation models, cell death has been attributed to a global insult, e.g., an elevation of the intracranial pressure resulting in global ischemia/hypoxia [19, 58, 59, 63]. However, several studies have shown that SAH produced by cautious blood injection can be also associated with neuronal apoptosis, independent of increased intracranial pressure [49, $50,68,82]$. In the absence of a global ischemic insult, the actual trigger for cell death is often uncertain [7].

Given the absence of a global insult, the absence of direct mechanical or hemorrhagic injury to the hippocampus, and the muted inflammatory response in the hippocampus, we were initially surprised to find apoptosis of granule cells of the dentate gyrus. Apoptosis of granule cells was characterized by pyknotic nuclei that stained with Fluoro-Jade and showed cleaved caspase-3. The fact that we were injuring the entorhinal cortex gave rise to the possibility that, if not attributable to a global insult or to direct injury, apoptosis might be due instead to a transsynaptic process. Transsynaptic degeneration and transsynaptic apoptosis have been well documented under a variety of conditions in many brain regions following deafferentation, including the system of entorhinal cortex-perforant pathway-dentate gyrus that we studied here $[27,40,71]$. Our findings of degeneration/demyelination of the perforant pathway, as indicated by Fluoro-Jade C staining [69], and diminished labeling with Black Gold II are consistent with deafferentation of dentate gyrus cells secondary to the robust inflammatory response in the entorhinal cortex. Injury to the perforant pathway that causes deafferentation of granule cells renders these cells susceptible to excitotoxic injury and apoptotic death [40]. Moreover, transsynaptic apoptosis is worsened by neuroinflammation [13], as we observed here. Importantly, demyelination and transsynaptic apoptosis in our model were largely blocked by delayed IV infusion of low-dose heparin.

To our knowledge, transsynaptic apoptosis has not been previously identified in SAH. The potential implications of transsynaptic apoptosis in SAH may be large, especially if $\mathrm{SAH}$ involves the eloquent cortex. Distinct from generally recognized triggers of apoptosis, such as direct tissue trauma, ischemia, inflammation, etc., transsynaptic apoptosis involves the spread of cell death beyond the boundaries of the immediate injury to involve otherwise uninjured neurons that are an axon's length away. This phenomenon may help to explain the prevalence of cognitive and neuropsychological deficits that commonly afflict humans with "mild-tomoderate" SAH who do not suffer a global insult $[6,10]$.

In summary, our findings here support previous work showing a robust neuroinflammatory response in the cortex underlying $\mathrm{SAH}$ which can lead to nearby tissue damage and cell death, as well as remote transsynaptic apoptosis. Our studies also show that delayed IV infusion of low-dose heparin exerts a powerful anti-inflammatory effect that lessens tissue injury and reduces transsynaptic apoptosis.

Acknowledgments This work was supported by grants to JMS from the National Institute of Neurological Disorders and Stroke (NS061808) and the National Heart, Lung and Blood Institute (HL082517).

Open Access This article is distributed under the terms of the Creative Commons Attribution License which permits any use, distribution and reproduction in any medium, provided the original author(s) and source are credited.

\section{References}

1. Amiconi G, Zolla L, Vecchini P, et al. The effect of macromolecular polyanions on the functional properties of human hemoglobin. Eur J Biochem. 1977;76:339-43.

2. Ayer R, Jadhav V, Sugawara T, et al. The neuroprotective effects of cyclooxygenase- 2 inhibition in a mouse model of aneurysmal subarachnoid hemorrhage. Acta Neurochir Suppl. 2011;111:145-9.

3. Bauer J, Sminia T, Wouterlood FG, et al. Phagocytic activity of macrophages and microglial cells during the course of acute and 
chronic relapsing experimental autoimmune encephalomyelitis. J Neurosci Res. 1994;38:365-75.

4. Berry CN, Girard D, Lochot S, et al. Antithrombotic actions of argatroban in rat models of venous, 'mixed' and arterial thrombosis, and its effects on the tail transection bleeding time. $\mathrm{Br} \mathrm{J}$ Pharmacol. 1994;113:1209-14.

5. Bhardwaj A. Molecular targets for ameliorating early brain injury post subarachnoid hemorrhage: a new focus. Crit Care Med. 2010;38:727-8.

6. Buchanan KM, Elias LJ, Goplen GB. Differing perspectives on outcome after subarachnoid hemorrhage: the patient, the relative, the neurosurgeon. Neurosurgery. 2000;46:831-8.

7. Cahill J, Calvert JW, Zhang JH. Mechanisms of early brain injury after subarachnoid hemorrhage. J Cereb Blood Flow Metab. 2006;26:1341-53.

8. Cahill J, Zhang JH. Subarachnoid hemorrhage: is it time for a new direction? Stroke. 2009;40:S86-7.

9. Casu B. Structure of heparin and heparin fragments. Ann N Y Acad Sci. 1989;556:1-17.

10. Chahal N, Barker-Collo S, Feigin V. Cognitive and functional outcomes of 5-year subarachnoid haemorrhage survivors: comparison to matched healthy controls. Neuroepidemiology. 2011; 37:31-8.

11. Chaichana KL, Pradilla G, Huang J, et al. Role of inflammation (leukocyte-endothelial cell interactions) in vasospasm after subarachnoid hemorrhage. World Neurosurg. 2010;73:22-41.

12. Chansel D, Ciroldi M, Vandermeersch S, et al. Heparin binding EGF is necessary for vasospastic response to endothelin. FASEB J. 2006;20:1936-8.

13. Chew DJ, Carlstedt T, Shortland PJ. A comparative histological analysis of two models of nerve root avulsion injury in the adult rat. Neuropathol Appl Neurobiol. 2011;37:613-32.

14. Coombe DR. Biological implications of glycosaminoglycan interactions with haemopoietic cytokines. Immunol Cell Biol. 2008;86:598-607.

15. Dijkstra CD, Dopp EA, Joling P, et al. The heterogeneity of mononuclear phagocytes in lymphoid organs: distinct macrophage subpopulations in the rat recognized by monoclonal antibodies ED1, ED2 and ED3. Immunology. 1985;54:589-99.

16. Dinarello CA. A clinical perspective of IL-1beta as the gatekeeper of inflammation. Eur J Immunol. 2011;41:1203-17.

17. Dumont AS, Dumont RJ, Chow MM, et al. Cerebral vasospasm after subarachnoid hemorrhage: putative role of inflammation. Neurosurgery. 2003;53:123-33.

18. Elsayed E, Becker RC. The impact of heparin compounds on cellular inflammatory responses: a construct for future investigation and pharmaceutical development. J Thromb Thrombolysis. 2003;15:11-8.

19. Endo H, Nito C, Kamada H, et al. Reduction in oxidative stress by superoxide dismutase overexpression attenuates acute brain injury after subarachnoid hemorrhage via activation of Akt/glycogen synthase kinase-3beta survival signaling. J Cereb Blood Flow Metab. 2007;27:975-82.

20. Engelberg H. Actions of heparin that may affect the malignant process. Cancer. 1999;85:257-72.

21. Etminan N, Vergouwen MD, Ilodigwe D, et al. Effect of pharmaceutical treatment on vasospasm, delayed cerebral ischemia, and clinical outcome in patients with aneurysmal subarachnoid hemorrhage: a systematic review and meta-analysis. J Cereb Blood Flow Metab. 2011;31:1443-51.

22. Friedrich V, Flores R, Muller A, et al. Reduction of neutrophil activity decreases early microvascular injury after subarachnoid haemorrhage. J Neuroinflammation. 2011;8:103.

23. Fryer A, Huang YC, Rao G, et al. Selective O-desulfation produces nonanticoagulant heparin that retains pharmacological activity in the lung. J Pharmacol Exp Ther. 1997;282:208-19.
24. Gallia GL, Tamargo RJ. Leukocyte-endothelial cell interactions in chronic vasospasm after subarachnoid hemorrhage. Neurol Res. 2006;28:750-8.

25. Gandhi NS, Mancera RL. The structure of glycosaminoglycans and their interactions with proteins. Chem Biol Drug Des. 2008;72:455-82.

26. Gerzanich V, Ivanov A, Ivanova S, et al. Alternative splicing of cGMP-dependent protein kinase I in angiotensin-hypertension: novel mechanism for nitrate tolerance in vascular smooth muscle. Circ Res. 2003;93:805-12.

27. Ginsberg SD, Martin LJ. Axonal transection in adult rat brain induces transsynaptic apoptosis and persistent atrophy of target neurons. J Neurotrauma. 2002;19:99-109.

28. Graeber MB, Lopez-Redondo F, Ikoma E, et al. The microglia/ macrophage response in the neonatal rat facial nucleus following axotomy. Brain Res. 1998;813:241-53.

29. Hanafy KA, Morgan SR, Fernandez L, et al. Cerebral inflammatory response and predictors of admission clinical grade after aneurysmal subarachnoid hemorrhage. J Clin Neurosci. 2010;17:22-5.

30. Hanel RA, Xavier AR, Mohammad Y, et al. Outcome following intracerebral hemorrhage and subarachnoid hemorrhage. Neurol Res. 2002;24 Suppl 1:S58-62.

31. Hansen-Schwartz J, Vajkoczy P, Macdonald RL, et al. Cerebral vasospasm: looking beyond vasoconstriction. Trends Pharmacol Sci. 2007;28:252-6.

32. Hiebert LM, Wice SM, McDuffie NM, et al. The heparin target organ - the endothelium. Studies in a rat model. Q J Med. 1993;86:341-8.

33. Higashiyama S, Iwabuki H, Morimoto C, et al. Membraneanchored growth factors, the epidermal growth factor family: beyond receptor ligands. Cancer Sci. 2008;99:214-20.

34. Higashiyama S, Nanba D. ADAM-mediated ectodomain shedding of HB-EGF in receptor cross-talk. Biochim Biophys Acta. 2005;1751:110-7.

35. Hirano K, Hirano M. Current perspective on the role of the thrombin receptor in cerebral vasospasm after subarachnoid hemorrhage. J Pharmacol Sci. 2010;114:127-33.

36. Hirsh J, Anand SS, Halperin JL, et al. Mechanism of action and pharmacology of unfractionated heparin. Arterioscler Thromb Vasc Biol. 2001;21:1094-6.

37. Ishikawa M, Kusaka G, Yamaguchi N, et al. Platelet and leukocyte adhesion in the microvasculature at the cerebral surface immediately after subarachnoid hemorrhage. Neurosurgery. 2009;64:546-53.

38. Johnston SC, Selvin S, Gress DR. The burden, trends, and demographics of mortality from subarachnoid hemorrhage. Neurology. 1998;50:1413-8.

39. Kalmes A, Daum G, Clowes AW. EGFR transactivation in the regulation of SMC function. Ann N Y Acad Sci. 2001;947:42-54.

40. Kovac AD, Kwidzinski E, Heimrich B, et al. Entorhinal cortex lesion in the mouse induces transsynaptic death of perforant path target neurons. Brain Pathol. 2004;14:249-57.

41. Kuwahara-Watanabe K, Hidai $\mathrm{C}$, Ikeda $\mathrm{H}$, et al. Heparin regulates transcription of endothelin-1 gene in endothelial cells. J Vasc Res. 2005;42:183-9.

42. Laohaprasit V, Mayberg MR. Risks of anticoagulation therapy after experimental corticectomy in the rat. Neurosurgery. 1993;32:625-8.

43. Lee JY, Keep RF, He Y, et al. Hemoglobin and iron handling in brain after subarachnoid hemorrhage and the effect of deferoxamine on early brain injury. J Cereb Blood Flow Metab. 2010;30:1793-803.

44. Lever R, Page CP. Novel drug development opportunities for heparin. Nat Rev Drug Discov. 2002;1:140-8.

45. Lindahl U, Lidholt K, Spillmann D, et al. More to "heparin" than anticoagulation. Thromb Res. 1994;75:1-32. 
46. Ling Y, Yang ZY, Yin T, et al. Heparin changes the conformation of high-mobility group protein 1 and decreases its affinity toward receptor for advanced glycation endproducts in vitro. Int Immunopharmacol. 2011;11:187-93.

47. Liu R, Mori S, Wake H, et al. Establishment of in vitro binding assay of high mobility group box-1 and S100A12 to receptor for advanced glycation endproducts: heparin's effect on binding. Acta Med Okayama. 2009;63:203-11.

48. Macdonald RL, Higashida RT, Keller E, et al. Clazosentan, an endothelin receptor antagonist, in patients with aneurysmal subarachnoid haemorrhage undergoing surgical clipping: a randomised, double-blind, placebo-controlled phase 3 trial (CONSCIOUS-2). Lancet Neurol. 2011;10:618-25.

49. Matz PG, Copin JC, Chan PH. Cell death after exposure to subarachnoid hemolysate correlates inversely with expression of CuZn-superoxide dismutase. Stroke. 2000;31:2450-9.

50. Matz PG, Fujimura M, Chan PH. Subarachnoid hemolysate produces DNA fragmentation in a pattern similar to apoptosis in mouse brain. Brain Res. 2000;858:312-9.

51. Mulloy B. The specificity of interactions between proteins and sulfated polysaccharides. An Acad Bras Cienc. 2005;77:651-64.

52. Murakami K, Koide M, Dumont TM, et al. Subarachnoid hemorrhage induces gliosis and increased expression of the proinflammatory cytokine high mobility group box 1 protein. Transl Stroke Res. 2011;2:72-9.

53. Myint KM, Yamamoto Y, Doi T, et al. RAGE control of diabetic nephropathy in a mouse model: effects of RAGE gene disruption and administration of low-molecular weight heparin. Diabetes. 2006;55:2510-22.

54. Nau R, Haase S, Bunkowski S, et al. Neuronal apoptosis in the dentate gyrus in humans with subarachnoid hemorrhage and cerebral hypoxia. Brain Pathol. 2002;12:329-36.

55. Niikawa S, Kitajima H, Ohe N, et al. Significance of acute cerebral swelling in patients with sylvian hematoma due to ruptured middle cerebral artery aneurysm, and its management. Neurol Med Chir (Tokyo). 1998;38:844-8

56. Nomura Y, Kawaguchi M, Yoshitani K, et al. Retrospective analysis of predictors of cerebral vasospasm after ruptured cerebral aneurysm surgery: influence of the location of subarachnoid blood. J Anesth. 2010;24:1-6.

57. Olson ST, Bjork I, Sheffer R, et al. Role of the antithrombinbinding pentasaccharide in heparin acceleration of antithrombinproteinase reactions. Resolution of the antithrombin conformational change contribution to heparin rate enhancement. J Biol Chem. 1992;267:12528-38.

58. Ostrowski RP, Colohan AR, Zhang JH. Mechanisms of hyperbaric oxygen-induced neuroprotection in a rat model of subarachnoid hemorrhage. J Cereb Blood Flow Metab. 2005;25:554-71.

59. Park S, Yamaguchi M, Zhou C, et al. Neurovascular protection reduces early brain injury after subarachnoid hemorrhage. Stroke. 2004;35:2412-7.

60. Patel AD, Gerzanich V, Geng Z, et al. Glibenclamide reduces hippocampal injury and preserves rapid spatial learning in a model of traumatic brain injury. J Neuropathol Exp Neurol. 2010;69:1177-90.

61. Pradilla G, Chaichana KL, Hoang S, et al. Inflammation and cerebral vasospasm after subarachnoid hemorrhage. Neurosurg Clin N Am. 2010;21:365-79.

62. Provencio JJ, Fu X, Siu A, et al. CSF neutrophils are implicated in the development of vasospasm in subarachnoid hemorrhage. Neurocrit Care. 2010;12:244-51.

63. Prunell GF, Svendgaard NA, Alkass K, et al. Delayed cell death related to acute cerebral blood flow changes following subarachnoid hemorrhage in the rat brain. J Neurosurg. 2005;102:1046-54.

64. Prunell GF, Svendgaard NA, Alkass K, et al. Inflammation in the brain after experimental subarachnoid hemorrhage. Neurosurgery. 2005;56:1082-92.
65. Rao NV, Argyle B, Xu X, et al. Low anticoagulant heparin targets multiple sites of inflammation, suppresses heparin-induced thrombocytopenia, and inhibits interaction of RAGE with its ligands. Am J Physiol Cell Physiol. 2010;299:C97-110.

66. Rider CC. Heparin/heparan sulphate binding in the TGF-beta cytokine superfamily. Biochem Soc Trans. 2006;34:458-60.

67. Robinson MJ, Tessier P, Poulsom R, et al. The S100 family heterodimer, MRP-8/14, binds with high affinity to heparin and heparan sulfate glycosaminoglycans on endothelial cells. J Biol Chem. 2002;277:3658-65.

68. Sabri M, Kawashima A, Ai J, et al. Neuronal and astrocytic apoptosis after subarachnoid hemorrhage: a possible cause for poor prognosis. Brain Res. 2008;1238:163-71.

69. Savaskan NE, Eyupoglu IY, Brauer AU, et al. Entorhinal cortex lesion studied with the novel dye Fluoro-Jade. Brain Res. 2000;864:44-51.

70. Schmued LC, Stowers CC, Scallet AC, et al. Fluoro-Jade C results in ultra high resolution and contrast labeling of degenerating neurons. Brain Res. 2005;1035:24-31.

71. Scholz J, Broom DC, Youn DH, et al. Blocking caspase activity prevents transsynaptic neuronal apoptosis and the loss of inhibition in lamina II of the dorsal horn after peripheral nerve injury. J Neurosci. 2005;25:7317-23.

72. Sercombe R, Dinh YR, Gomis P. Cerebrovascular inflammation following subarachnoid hemorrhage. Jpn J Pharmacol. 2002;88:227-49.

73. Simard JM, Geng Z, Woo SK, et al. Glibenclamide reduces inflammation, vasogenic edema, and caspase-3 activation after subarachnoid hemorrhage. J Cereb Blood Flow Metab. 2009;29:317-30.

74. Simard JM, Schreibman D, Aldrich EF, et al. Unfractionated heparin: multitargeted therapy for delayed neurological deficits induced by subarachnoid hemorrhage. Neurocrit Care. 2010;13:439-49.

75. Simard JM, Yurovsky V, Tsymbalyuk N, et al. Protective effect of delayed treatment with low-dose glibenclamide in three models of ischemic stroke. Stroke. 2009;40:604-9.

76. Smith KJ, Kapoor R, Felts PA. Demyelination: the role of reactive oxygen and nitrogen species. Brain Pathol. 1999;9:69-92.

77. Sozen T, Tsuchiyama R, Hasegawa Y, et al. Role of interleukin1beta in early brain injury after subarachnoid hemorrhage in mice. Stroke. 2009;40:2519-25.

78. Sozen T, Tsuchiyama R, Hasegawa Y, et al. Immunological response in early brain injury after SAH. Acta Neurochir Suppl. 2011;110:57-61.

79. Thourani VH, Brar SS, Kennedy TP, et al. Nonanticoagulant heparin inhibits NF-kappaB activation and attenuates myocardial reperfusion injury. Am J Physiol Heart Circ Physiol. 2000;278:H2084-93.

80. Tyrell DJ, Kilfeather S, Page CP. Therapeutic uses of heparin beyond its traditional role as an anticoagulant. Trends Pharmacol Sci. 1995;16:198-204.

81. Wang L, Brown JR, Varki A, et al. Heparin's anti-inflammatory effects require glucosamine 6-O-sulfation and are mediated by blockade of L- and P-selectins. J Clin Invest. 2002;110:127-36.

82. Wang L, Shi JX, Yin HX, et al. The influence of subarachnoid hemorrhage on neurons: an animal model. Ann Clin Lab Sci. 2005;35:79-85.

83. Weiler JM, Edens RE, Linhardt RJ, et al. Heparin and modified heparin inhibit complement activation in vivo. J Immunol. 1992;148:3210-5.

84. Wu Y, Tang K, Huang RQ, et al. Therapeutic potential of peroxisome proliferator-activated receptor gamma agonist rosiglitazone in cerebral vasospasm after a rat experimental subarachnoid hemorrhage model. J Neurol Sci. 2011;305:85-91.

85. Yokokawa K, Mandal AK, Kohno M, et al. Heparin suppresses endothelin-1 action and production in spontaneously hypertensive rats. Am J Physiol. 1992;263:R1035-41.

86. Young E. The anti-inflammatory effects of heparin and related compounds. Thromb Res. 2008;122:743-52. 